硼亲和吸附剂的制备及其对苯甲酰艮类农药的萃取性能

\author{
张 咏, 梅 萌, 刘 袜, 喻 杰, 黄晓佳 *, 袁东星 \\ (厦门大学环境与生态学院, 滨海湿地生态系统教育部重点实验室, 福建 厦门 361005)
}

摘要: 以 3-丙烯酰胺苯硼酸 (APB) 为单体, 二乙烯基苯 (DVB) 为交联剂, “原位” 聚合制备了聚( 3-丙烯酰胺苯硼酸二乙烯基苯) 多孔嗍亲和整体材料并作为搅拌饼固相萃取 (SCSE-APBDVB) 的萃取介质。以 5 种苯甲酰脲农药为 目标化合物, 详细考察了萃取过程中解吸溶剂、样品基底中 $\mathrm{pH}$ 值以及离子强度、萃取和解吸时间等实验条件对萃 取效率的影响。在此基础上, 与高效液相色谱-二极管阵列检测器联用建立了环境水样和果汁样品中苯甲酰䐂农 药残留的测定方法。在最佳条件下, 在水样和果汁样品中, 5 种目标化合物的检出限 (LOD, $S / N=3$ ) 分别在 0.055 $\sim 0.11 \mu \mathrm{g} / \mathrm{L}$ 和 $0.095 \sim 0.31 \mu \mathrm{g} / \mathrm{L}$ 之间, 所建立的方法具有理想的日内和日间重现性 ( RSD 值均小于 $9.0 \%$ )。在 对实际环境水样和果汁样品的测定中, 不同加标浓度苯甲酰䐂的回收率为 75.6\% 109\%。研究表明, 由于所制备 吸附剂与目标化合物存在 B-N 配位作用、氢键和疏水等多种作用力, 因此 SCSE-APBDVB 可对苯甲酰䐂农药进行 有效萃取,所建立的分析方法具有简便、灵敏和环境友好等特点。

关键词: 嗍亲和吸附剂; 摚拌饼固相萃取; 整体材料; 高效液相色谱; 苯甲酰腿农药; 富集

中图分类号: O658 文献标识码:A 文章编号: 1000-8713(2014)09-0981-07

\title{
Preparation of boronate affinity sorbent and its extraction performance for benzoylurea pesticides
}

\author{
ZHANG Yong, MEI Meng, LIU Yi, YU Jie, HUANG Xiaojia* , YUAN Dongxing \\ (Key Laboratory of the Ministry of Education for Coastal and Wetland Ecosystem, College of \\ the Environment and Ecology, Xiamen University, Xiamen 361005, China)
}

\begin{abstract}
A new boronate affinity monolithic material was prepared and used as the extraction medium of stir cake sorptive extraction (SCSE). The porous boronate affinity sorbent was prepared by in situ copolymerization of 3-acrylamidophenylboronic acid (APB) and divinyl benzene (DVB). To achieve optimum extraction performance for benzoylurea pesticides, several parameters, including desorption solvent, $\mathrm{pH}$ value, ionic strength in sample matrix, extraction and desorption times, were investigated in detail. At the same time, a simple, sensitive and environment friendly method for the determination of benzoylurea pesticides in water and juice samples was developed by the combination of SCSE-APBDVB with HPLC equipped with a diode array detector. Under the optimized experimental conditions, the limits of detection $(S / N=3)$ for target analytes were $0.055-0.11 \mu \mathrm{g} / \mathrm{L}$ in water and $0.095-0.31 \mu \mathrm{g} / \mathrm{L}$ in juice. The precision of the proposed method was evaluated in terms of intra- and inter-assay repeatability calculated as RSD, and it was found that the RSDs were all below 9. $0 \%$. The developed method was successfully applied to the determination of benzoylurea pesticide residues in water and juice samples and satisfactory recoveries of spiked target compounds were in the range of $75.6 \%-109 \%$. The results well demonstrate that the new sorbent can extract benzoylurea pesticides effectively through multi-interactions including boron-nitrogen coordination, hydrogen-bond and hydrophobic interactions between sorbent and analytes.
\end{abstract}

Key words : boronate affinity sorbent; stir cake sorptive extraction; monolithic material; high

*通讯联系人.Tel:( 0592) 2189278,E-mail:hxj@xmu.edu.cn.

基金项目:国家自然科学基金项目(21377105); 中央高校基本科研业务费(20720140510); 厦门大学本科生基础科研创新项目 ( CXB2013030, CXB2014006).

收稿日期: 2014-07-02 
performance liquid chromatography (HPLC) ; benzoylurea pesticides ; enrichment

自 20 世纪 80 年代以来,嗍亲和色谱(BAC) 已 经被广泛用于含顺式二羟基化合物如糖蛋白、核苷 等生物分子的选择性分离富集 ${ }^{[1,2]}$ 。其原理是基于 在碱性条件下, 嗍亲和介质与顺式二差基化合物形 成五元或六元环状酯类物质; 而在酸性条件下,该酯 类物质会发生解离, 释放出原来的物质从而达到选 择分离富集的目的。值得注意的是 BAC 中的嗍酸 配体很容易与氮原子形成 B-N 配合物。刘震等 ${ }^{[3]}$ 利用该特性,将间氨基苯硼酸、已二胺和三 (2,3-环 氧基丙基) 异丙氨酸酯( TEPIC) 共聚制备嗍亲和整 体柱, 研究表明, B-N 配合键的形成有利于 TEPIC 的开环聚合。我们也曾以 4-乙烯基苯硼酸为单体 制备了硼亲和吸附剂, 研究发现所制备的吸附剂对 一些含氮污染物如磺胺类、硝基酚等物质具有较好 的萃取性能 ${ }^{[4]}$ 。

苯甲酰脲类农药 (BUPs) 是一种昆虫生长调节 剂, 主要通过抑制昆虫蜕皮、变态, 导致不育及直接 杀卵等方面的作用 ${ }^{[5]}$ 来控制害虫, 目前 BUPs 在粮 食、蔬菜、水果生产中有广泛的应用,但其残留对人 体和环境存在潜在的危险。我国规定这类农药在蔬 菜和水果中的最大允许残留量在 $0.5 \sim 3.0 \mathrm{mg} / \mathrm{kg}$ 之间 ${ }^{[6]}$, 因此建立高效、灵敏的 BUPs 检测方法具有 重要意义。液相色谱是目前测定 BUPs 的常用方 法,但在进行色谱分离检测前需进行必要的样品前 处理以实现对目标物的富集和去除样品基底的干 扰。目前,测定 BUPs 的前处理方法包括液液萃 取 ${ }^{[7]}$ 、分散液液萃取 ${ }^{[8-10]}$ 、单滴微萃取 ${ }^{[6]}$ 和固相萃 取 ${ }^{[11]}$, 但这些方法存在着诸如需要较多的有机溶 剂、富集容量较低及操作过程繁琐等不足。因此,发 展操作简便、萃取容量高和环境友好的萃取方式对 于 BUPs 残留监测具有重要的现实意义。

搅拌饼固相萃取 (SCSE) 是由我们研究小组自 行设计、制作的一种新型样品前处理方法, 其集萃 取、富集和净化为一体, 具有操作简便、使用灵活和 环境友好等特点 ${ }^{[12-14]}$ 。同时, 与传统的搅拌棒固相 萃取 (SBSE) 相比, 由于 SCSE 在使用过程中, 萃取 介质不与容器壁接触, 不会产生磨损, 因此具有理想 的使用寿命。本研究以 3-丙烯酰胺苯硼酸 (APB) 为单体, 二乙烯基苯 (DVB) 为交联剂, 利用整体材 料的“原位” 聚合特点制备了嗍亲和吸附剂并作为 SCSE 的萃取介质。同时与高效液相色谱-二极管阵 列检测器 (HPLC/DAD) 联用, 建立可对环境水样和 果汁样品中 BUPs 残留进行有效监测的 SCSE-
APBDVB-HPLC/DAD 分离分析体系。本研究有望 为水样和果汁样品中苯甲酰脲类农药残留监测提供 一种简便、灵敏和环境友好的分析方法。

\section{1 实验部分}

\section{1 仪器与试剂}

高效液相色谱设备: LC-20AB 泵, CBA-20A 控 制器, SPD-M20A DAD 检测器 ( Shimadzu, Kyoto, Japan )，7725i 进样阀 (Rheodyne, USA); $20 \mu \mathrm{L}$ 定量阀。材料的扫描电镜表征在 Model XL30 电镜 仪上完成(Philips, Eindhoven, The Netherlands)。

3-丙烯酰胺苯硼酸 (APB, 纯度 $98 \%$ ), 购自苏州 苏凯路化学科技有限公司; 二乙烯基苯 (DVB, 纯度 $85 \%$ ) 由 Alfa Aesar 公司 (天津) 提供; 二甲基亚砜 (DMSO)（分析纯）,购自天津市光复精细化工研究 所。5 种苯甲酰艮类农药氟铃脲 ( HFU)、氟苯脲 $(T F U) 、$ 風螨脲 $(\mathrm{LFU})$ 、氟虫脲 $(\mathrm{FFU})$ 和氟啶腿 (CFU) 的标准品 (其化学结构如图 1), 购自北京振 翔工贸有限责任公司。全部实验用水均为超纯水 (Millipore, Bedford, MA，USA), 实验中的其他试 剂均为分析纯。实际环境水样采自厦门的湖水和农 田水,果汁样品购自厦门当地超市。

\section{2 标准溶液的配制}

分别称取 $5.0 \mathrm{mg}$ 各标准品, 用甲醇溶解并定 容于 $50 \mathrm{~mL}$ 棕色瓶中, 配制成 $100 \mathrm{mg} / \mathrm{L}$ 的单标准 储备液, 置于 $4{ }^{\circ} \mathrm{C}$ 的冰箱中保存。使用时, 取各单标 准储备液适量, 用甲醇配制成所需浓度的混合标准 溶液。

\section{3 色谱条件}

色谱分离柱: Kromasil LC-18 柱( $250 \mathrm{~mm} \times 4.6$ $\mathrm{mm}, 5 \mu \mathrm{m})$; 流动相: 水/乙腈 $(22 / 78, \mathrm{v} / \mathrm{v})$, 等度 洗脱; 流速: $1.0 \mathrm{~mL} / \mathrm{min}$; 检测波长: $260 \mathrm{~nm}$; 进样体 积: $20 \mu \mathrm{L}$

\subsection{SCSE-APBDVB 的制备}

SCSE-APBDVB 的制备过程非常简单, 主要包 括 3 个步骤。

(1) poly (APB-DVB) 萃取饼的制备: 将单体 $\mathrm{APB}$ 、交联剂 DVB (单体和交联剂合称为单体混合 物 ）、致孔剂 DMSO 和引发剂 $\mathrm{AIBN}$ (总量的 $1 \%, \mathrm{w} /$ w) 按一定比例混合 (单体和交联剂的质量比以及单 体混合物和致孔剂的质量比均为 60/40) 并超声至 完全溶解,通氮气 $3 \mathrm{~min}$ 除氧,然后将该聚合反应溶 液加人一端密封的一次性注射器中, 并密封另一端, 


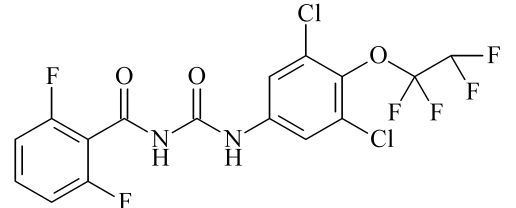

Hexaflumuron

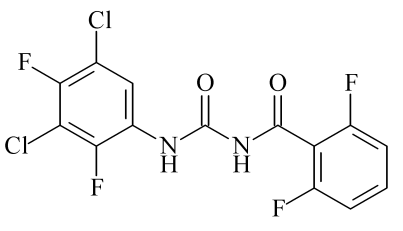

Teflubenzuron

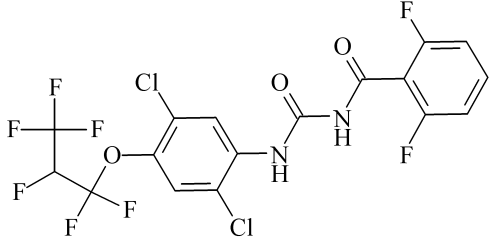

Lufenuron

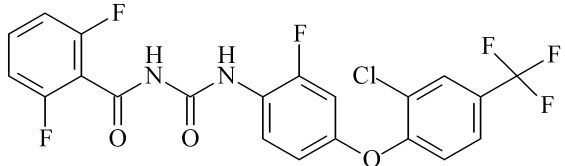

Flufenoxuron

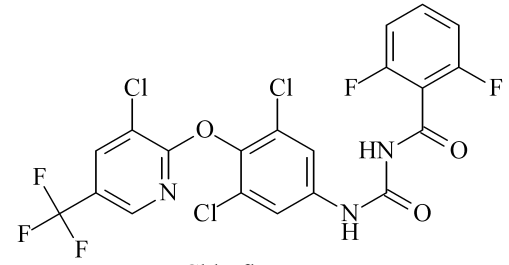

Chlorfluazuron

图 15 种苯甲酰脲类农药的分子结构式

Fig. 1 Molecular structures of five benzoylurea pesticides

保持注射器竖直, 放人烘箱中于 $70{ }^{\circ} \mathrm{C}$ 恒温反应 24 $\mathrm{h}$ 。最后,将整体材料萃取饼缓慢地从注射器中推 出,用甲醇多次浸泡去除致孔剂和未反应完全的单 体混合物。

(2) 搅拌磁子的制作:截取 $1.3 \mathrm{~cm}$ 铁丝 (直径 $1.0 \mathrm{~mm})$,清洗烘干后放人直径略大于铁丝的玻璃 毛细管中,然后将其两端熔融密封得摚拌磁子。再 将磁子放人甲醇中浸泡清洗后, 即可使用。

(3) 萃取饼支撑体的制作:截取一次性注射器 针筒部分约 $0.6 \mathrm{~cm}$ 长,在底端钻两个小孔,使搅拌 磁子贯穿其中,将另一端刻成锯齿状,同时在底端多 钻 6 个小孔, 以增加萃取介质与样品溶液的接触, 提 高传质通量。最后, 将萃取饼小心嵌人支撑体中, 即 得 SCSE-APBDVB。该过程可参考文献 ${ }^{[12,13]}$ 。

\section{5 实际样品前处理}

对于环境水样: 取 $100 \mathrm{~mL}$ 水样,过 $0.45 \mu \mathrm{m}$ 滤 膜, 将其 $\mathrm{pH}$ 值调为 7.0, 然后放人 SCSE-APBDB 以 $300 \mathrm{r} / \mathrm{min}$ 的速度进行摚拌萃取 $2.5 \mathrm{~h}$ 。萃取完成 后, 取出 SCSE-APBDB 放人 $3.0 \mathrm{~mL}$ 的甲醇 $/ 0.1 \%$ 甲酸水溶液 $(95 / 5, v / v)$ 中, 在同样的搅拌速度条件 下解吸 $0.5 \mathrm{~h}$, 解吸液用氮气吹干, 用 $0.5 \mathrm{~mL}$ 甲醇 定容后进行 HPLC 测定。

对于果汁样品: 将果汁在 $3500 \mathrm{r} / \mathrm{min}$ 的速度 下离心 $10 \mathrm{~min}$, 取上层溶液, 然后过 $1.0 \mu \mathrm{m}$ 滤膜。 取滤液 $5 \mathrm{~mL}$, 用超纯水稀释到 $100 \mathrm{~mL}$ 并调 $\mathrm{pH}$ 值 为 7.0 , 然后按环境水样的操作过程进行样品处理 和测定。

\section{2 结果与讨论}

2.1 SCSE-APBDB 的制备条件优化及电镜表征
通常情况下,单体、交联剂和致孔剂的比例会影 响整体材料的功能基团以及孔隙的结构, 从而影响 到吸附剂的萃取性能。为了获得最优的萃取性能, 我们对单体/交联剂比例及单体混合物/致孔剂比例 进行了考察,发现不同单体、交联剂和致孔剂用量对 吸附剂的吸附性能、材质硬度均有较大影响。综合 考虑萃取效果和使用寿命, 选择单体和交联剂的质 量比以及单体混合物与致孔剂的质量比均为 60/40 为最优制备条件。在该制备条件下, SCSE-APBD$\mathrm{VB}$ 具有良好的制备重现性, 对目标物的富集因子的 相对标准偏差 (RSD, $n=4$ ) 在 $10 \%$ 以下。同时 SCSE-APBDVB 具有理想的使用寿命, 至少可连续 使用 150 次以上。

图 2 是在最佳制备条件下,poly (APB-DVB) 整 体材料放大 10000 倍的电镜扫描图, 可清晰地看到 整体材料均一的孔隙结构和相互交联的骨架结构。

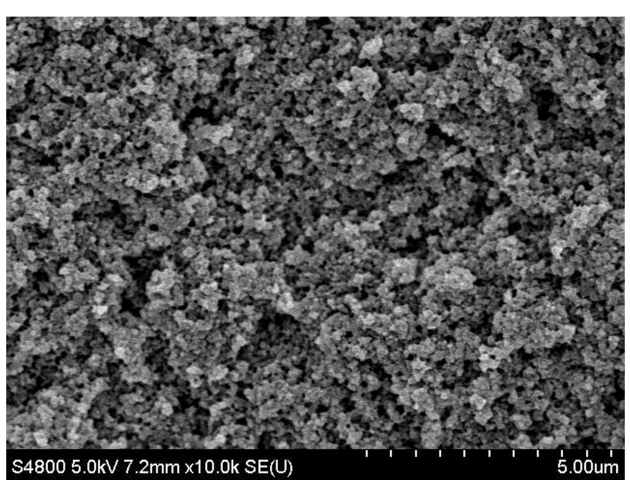

图 2 poly (APB-DVB) 整体材料的电镜图

Fig. 2 Scanning-electron micrograph of poly (APB-DVB) monolithic material

\section{2 萃取条件优化}

在 SCSE 过程中, 解吸溶剂、样品基底 $\mathrm{pH}$ 值和 
离子强度、萃取和解吸时间等因素对目标物的富集 性能有较大影响。为了得到 SCSE-APBDVB 对 5 种 BUPs 的最佳萃取条件,本研究对上述萃取条件进 行了详细优化。

\subsection{1 解吸溶剂的影响}

本研究以甲醇 $/ 0.1 \%$ 甲酸水溶液为解吸溶剂, 考察了甲醇含量对解吸效果的影响。从图 3 结果可 以看出, 当以甲醇 $/ 0.1 \%$ 甲酸水溶液 $(95 / 5, \mathrm{v} / \mathrm{v})$ 为 解吸溶剂时, 5 种目标物的解吸效率达到最大值。 这主要是因为所制备的嗍亲和吸附剂与目标物存在 着 B-N 配合、氢键和偶极-偶极等作用力,适量的酸 可破坏这些相互作用力, 因此有利于目标物的解吸。 根据实验结果, 选择甲醇 $/ 0.1 \%$ 甲酸水溶液 $(95 / 5$, $\mathrm{v} / \mathrm{v}$ ) 为解吸溶剂。

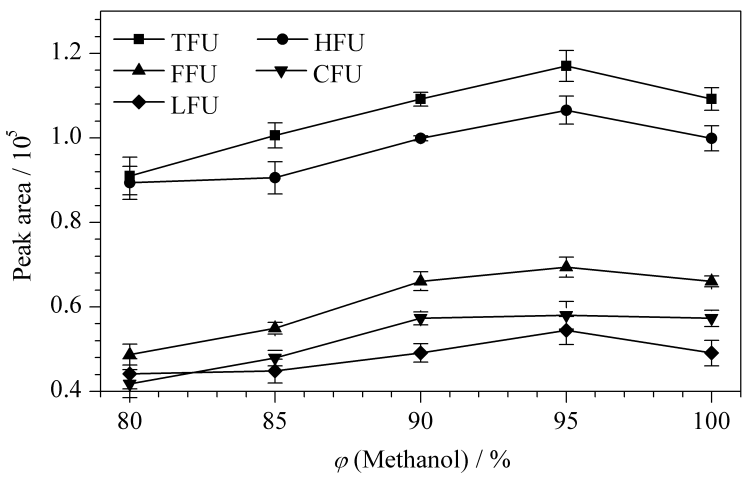

图 3 解吸溶剂中甲醇的体积分数对萃取效率的影响

Fig. 3 Effect of volume percentage of methanol in desorption solvent on extraction efficiency

Conditions: extraction and desorption times were both 1.0 $\mathrm{h}$; the $\mathrm{pH}$ value of sample matrix did not be adjusted; no salt was added; the spiked mass concentration of each analyte was $100 \mu \mathrm{g} / \mathrm{L}$

\subsection{2 $\mathrm{pH}$ 值的影响}

图 4 为样品基底 $\mathrm{pH}$ 值从 2 变化到 9 时,萃取 效率随 $\mathrm{pH}$ 值的变化曲线。可以看出,随着 $\mathrm{pH}$ 值的 升高,萃取效率缓慢上升 ( FFU 和 CFU 除外); 当 $\mathrm{pH}$ 升高到 7. 0 时, SCSE-APBDVB 对 5 种 BFUs 的 萃取效率达到最大值; $\mathrm{pH}$ 值继续增加时, 萃取效率 明显变小。该变化趋势的主要原因可能为:在低 $\mathrm{pH}$ 值时,BUFs 分子中 $\mathrm{N}$ 原子被质子化无法与吸附剂 产生 B-N 配合及氢键作用力, 此时吸附剂主要通过 疏水和 $\pi-\pi$ 作用吸附目标物。随着 $\mathrm{pH}$ 值的升高, 去质子化作用逐渐生成, 因此吸附剂和目标物之间 除了疏水和 $\pi-\pi$ 作用力外,还形成了 $\mathrm{B}-\mathrm{N}$ 配合及氢 键作用力, 多重作用力导致了萃取效率的提高。但 当 $\mathrm{pH}$ 值继续增加时 (高于 8.0 ), 吸附剂中的嗍酸 解离, B-N 配合作用力下降,因此导致萃取效率的减 少。根据实验结果, 选择 7.0 为最优 $\mathrm{pH}$ 值。

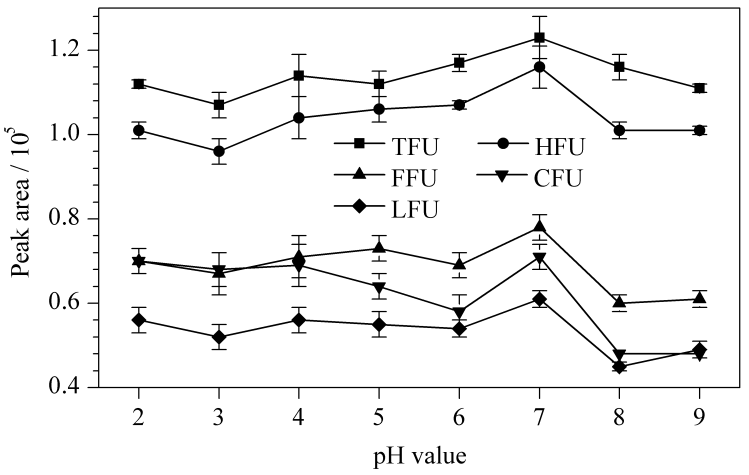

图 4 样品 $\mathrm{pH}$ 值对萃取效率的影响

Fig. 4 Effect of $\mathrm{pH}$ value of sample matrix on extraction efficiency

Conditions: methanol/0.1\% formic acid aqueous (95/5, v/ v) was selected as desorption solvent; the sample $\mathrm{pH}$ values were adjusted by $0.1 \mathrm{~mol} / \mathrm{L} \mathrm{HCl}$ or $0.1 \mathrm{~mol} / \mathrm{L} \mathrm{NaOH}$. The other conditions are the same as in Fig. 3.

\subsection{3 离子强度的影响}

根据文献 ${ }^{[15,16]}$, 对于极性化合物的萃取, 由于 同时有利于萃取的盐析作用及不利于萃取的静电相 互作用,因此离子强度对萃取效率有很大影响。本 实验通过添加不同量的 $\mathrm{NaCl} （ 0 、 50 、 100 、 150 、 200$ 、 250 和 $300 \mathrm{~g} / \mathrm{L})$ 来改变基底的离子强度。从图 5 可 以看出, 离子强度的增加不利于目标物质的萃取, 这 可能是因为随着离子强度的增加, 由于盐离子的影 响使目标物质与溶液间的静电作用力增强, 增加了 目标物在溶液中的溶解从而导致萃取效果的下降, 因此在后续实验中不调节样品的离子强度。

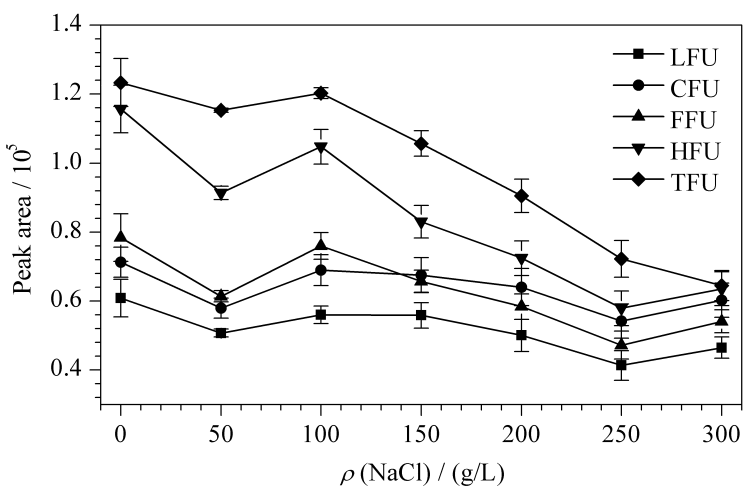

图 5 离子强度对萃取效率的影响

Fig. 5 Effect of ionic strength in sample matrix on extraction efficiency

Conditions: $\mathrm{pH}$ value was adjusted to 7.0. The other conditions are the same as in Fig. 4.

\subsection{4 萃取和解吸时间的影响}

在其他实验条件保持不变时,考察了萃取时间 在 $0.5 \sim 2.5 \mathrm{~h}$ 之间对萃取效果的影响。研究表明 SCSE-APBDVB 对 5 种 BUPs 的萃取效率随萃取时 间的增加而迅速上升, 在 $2.5 \mathrm{~h}$ 时, 萃取仍未达到平 
衡, 这说明所制备的吸附剂对目标物具有较强的萃 取性能。综合考虑分析时间及灵敏度,选择 $2.5 \mathrm{~h}$ 为最佳萃取时间。本研究还考察了解吸时间在 0.5 $\sim 2.0 \mathrm{~h}$ 之间解吸效果的变化, 实验结果表明在 0.5 $\mathrm{h}$ 的时候, 目标化合物可以从 SCSE-APBDVB 中被 完全解吸, 因此解吸时间确定为 $0.5 \mathrm{~h}$ 。

综合上述实验结果, SCSE-APBDVB 对 5 种 BUPs 的最佳萃取条件为: 甲醇 $/ 0.1 \%$ 甲酸水溶液 (95/5, v/v) 为解吸溶剂,基底 $\mathrm{pH}$ 值调为 7.0 ,不调 样品的离子强度, 吸附和解吸时间分别为 $2.5 \mathrm{~h}$ 和 $0.5 \mathrm{~h}$ 。

\section{3 方法的有效性}

在空白的水样和果汁样品中添加不同量的标准 储备液,配制不同浓度梯度的溶液, 在最佳的萃取条
件下, 考察方法的线性动力学范围、线性相关系数、 检出限、定量限及日内和日间重复性, 结果见表 1 。 可以看出, 在水样中, HFU 和 TFU 的线性范围为 $0.25 \sim 100.0 \mu \mathrm{g} / \mathrm{L}$, 其他 3 种 BUPs 的线性范围为 $0.50 \sim 100.0 \mu \mathrm{g} / \mathrm{L}, 5$ 种目标化合物均具有较好的 线性关系 $(r>0.98), \operatorname{LOD}(S / N=3)$ 在 $0.055 \sim 0.11$ $\mu \mathrm{g} / \mathrm{L}$ 之间, $\operatorname{LOQ}(S / N=10)$ 在 $0.18 \sim 0.38 \mu \mathrm{g} / \mathrm{L}$ 之 间。而对于果汁样品, HFU 和 TFU 的线性范围为 $0.50 \sim 100 \mu \mathrm{g} / \mathrm{L}$, 其他 3 种 BUPs 的线性范围为 1. $00 \sim 100.0 \mu \mathrm{g} / \mathrm{L}, 5$ 种目标化合物的线性关系良 好 $(r>0.99), \mathrm{LOD}$ 在 $0.095 \sim 0.31 \mu \mathrm{g} / \mathrm{L}$ 之间, $\mathrm{LOQ}$ 在 $0.31 \sim 1.00 \mu \mathrm{g} / \mathrm{L}$ 之间; 同时所建方法的日内和 日间的实验重复性良好 $(\mathrm{RSD}<9.0 \%)$; 表明本方法 具有较高的灵敏度和实验重复性。

表 15 种 BUPs 的线性动力学范围、线性相关系数、检出限、定量限及日内和日间重复性

Table 1 Linear dynamic ranges, correlation coefficients, LODs and LOQs, inter-day and intra-day precisions achieved for the five BUPs

\begin{tabular}{cccccccc}
\hline Sample & Compound & $\begin{array}{c}\text { Linear range/ } \\
(\mu \mathrm{g} / \mathrm{L})\end{array}$ & $r$ & $\begin{array}{c}\mathrm{LD}^{\mathrm{a}} / \\
(\mu \mathrm{g} / \mathrm{L})\end{array}$ & $\begin{array}{c}\mathrm{LOQ}^{\mathrm{b}} / \\
(\mu \mathrm{g} / \mathrm{L})\end{array}$ & $\begin{array}{c}\text { Intra-assay variability }^{\mathrm{c}} \\
(\mathrm{RSD} / \% ; n=3)\end{array}$ & $\begin{array}{c}\text { Inter-assay variability }^{\mathrm{c}} \\
(\mathrm{RSD} / \% ; n=4)\end{array}$ \\
\hline \multirow{2}{*}{ Water } & HFU & $0.25-100.0$ & 0.9996 & 0.055 & 0.18 & 4.22 & 4.72 \\
& TFU & $0.25-100.0$ & 0.9957 & 0.067 & 0.22 & 3.60 & 2.40 \\
& LFU & $0.50-100.0$ & 0.9812 & 0.11 & 0.38 & 3.62 & 6.95 \\
& FFU & $0.50-100.0$ & 0.9859 & 0.10 & 0.33 & 6.96 & 7.45 \\
& CFU & $0.50-100.0$ & 0.9806 & 0.11 & 0.36 & 4.01 & 7.36 \\
& HFU & $0.50-100.0$ & 0.9982 & 0.095 & 0.31 & 7.26 & 6.44 \\
& TFU & $0.50-100.0$ & 0.9989 & 0.096 & 0.32 & 6.20 & 8.93 \\
& LFU & $1.00-100.0$ & 0.9994 & 0.27 & 0.95 & 0.78 & 4.70 \\
& FFU & $1.00-100.0$ & 0.9974 & 0.25 & 0.81 & 3.14 & 6.01 \\
\hline
\end{tabular}

a. $S / N=3$; b. $S / N=10$; c. assays at $50 \mu \mathrm{g} / \mathrm{L}$ level.

\section{4 与现有的其他方法的比较}

表 2 列出了本研究所建立的方法与现有的测定 水样和果汁样品中 BUPs 的方法在 LOD 值和回收 率方面的比较。从数据可以看出, 对于水样, 在用紫 外检测器检测时, 本方法所得到的 LOD 值低于其他 方法所得 LOD 值 ${ }^{[8-10]}$; 同样的, 对于果汁样品, 本实
验得到的 LOD 值也低于以前研究所取得的 LOD 值 ${ }^{[5,6]}$ 。通常情况下, 利用质谱检测器可得到比紫 外检测器更高的灵敏度 ${ }^{[11]}$ 。但在测定果汁样品时, 本方法对 TFU 和 FFU 的灵敏度仍高于 LLE$\mathrm{HPLC} / \mathrm{MS} / \mathrm{MS}^{[7]}$ 。从表 1 中数据还可以看出本方 法所取得的加标回收率与以前的研究方法处于相同

表 2 所建方法与现有的测定水样和果汁样品中 BUPs 的方法在 LOD 值和回收率方面的比较

Table 2 Comparison of LODs and recoveries of present work with other methods based on sorbent extraction for the determination of BUPs

\begin{tabular}{|c|c|c|c|c|c|c|c|c|}
\hline \multirow{2}{*}{ Method * } & \multirow{2}{*}{ Sample } & \multicolumn{5}{|c|}{$\mathrm{LODs} /(\mu \mathrm{g} / \mathrm{L})$} & \multirow{2}{*}{ Recoveries $/ \%$} & \multirow{2}{*}{ Reference } \\
\hline & & $\mathrm{HFU}$ & $\mathrm{TFU}$ & LFU & FFU & $\mathrm{CFU}$ & & \\
\hline DLPME-LC/UV & water & $/$ & $/$ & / & 0.21 & 0.24 & $85.1-108.6$ & {$[8]$} \\
\hline DLPME-LC/UV & water & / & / & / & 0.63 & 0.62 & $72.4-103.5$ & {$[9]$} \\
\hline DLPME-LC/UV & water & 0.10 & 0.07 & / & 0.15 & 0.05 & $79.8-91.7$ & {$[10]$} \\
\hline SPE-HPLC/MS & water & 0.0042 & 0.0052 & 0.0075 & 0.0035 & / & $73.1-110.4$ & {$[11]$} \\
\hline SDME-LC/UV & juice & 10 & 10 & I & I & / & $83.5-80.8$ & {$[6]$} \\
\hline FDME-LC/UV & juice & 5 & 10 & / & / & / & $88.5-91.5$ & {$[5]$} \\
\hline LLE-HPLC/MS/MS & juice & 0.05 & 0.11 & 0.05 & 0.42 & 0.08 & $92.0-110$ & {$[7]$} \\
\hline SCSE-LC/DAD & water & 0.055 & 0.067 & 0.11 & 0.10 & 0.11 & $75.6-99.4$ & present work \\
\hline SCSE-LC/DAD & juice & 0.095 & 0.096 & 0.27 & 0.25 & 0.31 & $81.6-114$ & present work \\
\hline
\end{tabular}

* DLPME: dispersive liquid-phase microextraction; SPE: solid-phase microextraction; SDME: single-drop microextraction; FDME: floated organic drop microextraction; LLE: liquid-liquid microextraction. 
水平。

\section{5 实际样品测定及加标回收率实验}

将所建立的方法用于实际水样和果汁样品中 BUPs 的测定, 结果如表 3 所示, 图 6 为实际样品和 加标后的样品经 SCSE-APBDVB 萃取后的色谱图。 结果表明,在一环境水样中检测到低浓度的 HFU, 而其他的实际样品均未检出 BUPs。实际样品中复 杂基底可能对萃取效率产生影响, 为了考察所建立

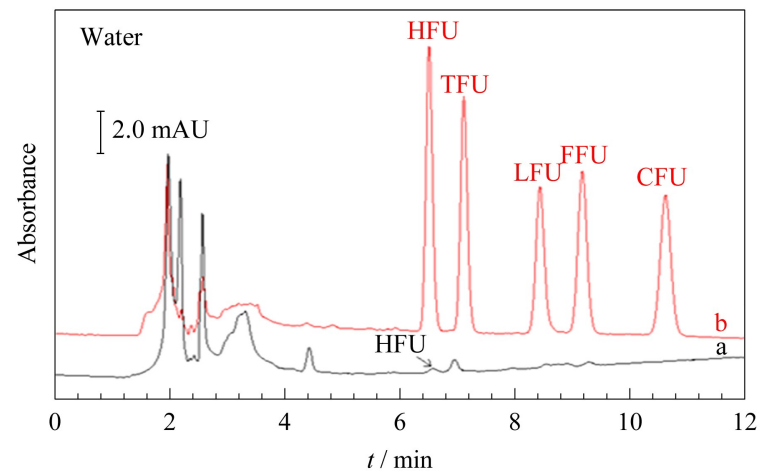

的方法用于实际样品中 5 种 BUPs 物质测定的可行 性, 测定实际水样和果汁样品中不同加标量的回收 率。从表 3 可以看出, 在不同实际水样和果汁样品 中的两种加标水平 $(5.0$ 和 $50.0 \mu \mathrm{g} / \mathrm{L})$ 下, 5 种目标 物均取得了理想的回收率, 在环境水样和果汁样品 中的回收率分别为 $75.6 \% \sim 99.4 \%$ 和 $81.6 \%$ $114 \%$ 。研究结果表明所建立的方法对于环境水样 和果汁样品中 BUPs 的检测具有较好的可行性。

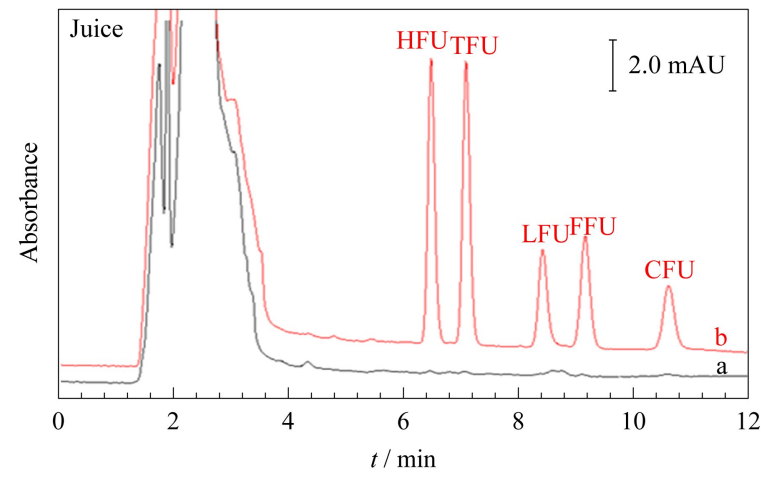

图 6 实际水样和果汁样品经 SCSE-APBDVB 萃取后的色谱图

Fig. 6 HPLC chromatograms of water and juice samples after enrichment with SCSE-APBDVB

a. un-spiked samples treated with SCSE-APBDVB; b. spiked samples with each analyte at $50 \mu \mathrm{g} / \mathrm{L}$ and treated with SCSE-APBDVB.

Conditions: methanol/0. 1\% formic acid aqueous $(95 / 5, \mathrm{v} / \mathrm{v})$ as desorption solvent; the sample pH values were adjusted to 7.0 ; no salt was added; the extraction and desorption times were 2.5 and $0.5 \mathrm{~h}$, respectively.

表 3 实际水样和果汁样品中 5 种目标物的测定结果及加标回收率

Table 3 Results of determination and spiked recoveries of the five target analytes in water and juice samples

\begin{tabular}{|c|c|c|c|c|c|c|c|c|c|c|c|c|c|c|c|}
\hline \multirow{2}{*}{ Sample } & \multicolumn{5}{|c|}{ Spiked/( $\mu \mathrm{g} / \mathrm{L})$} & \multicolumn{5}{|c|}{ Detected/ $(\mu \mathrm{g} / \mathrm{L})$} & \multicolumn{5}{|c|}{ Recoveries/\% } \\
\hline & $\mathrm{HFU}$ & TFU & $\mathrm{LFU}$ & $\mathrm{FFU}$ & $\mathrm{CFU}$ & $\mathrm{HFU}$ & TFU & LFU & FFU & $\mathrm{CFU}$ & $\mathrm{HFU}$ & TFU & LFU & $\mathrm{FFU}$ & $\mathrm{CFU}$ \\
\hline \multicolumn{16}{|l|}{ Water } \\
\hline \multirow[t]{3}{*}{ Sample 1} & 0 & 0 & 0 & 0 & 0 & 0.25 & $\mathrm{ND}$ & $\mathrm{ND}$ & ND & $\mathrm{ND}$ & & & & & \\
\hline & 5.0 & 5.0 & 5.0 & 5.0 & 5.0 & 4.03 & 4.01 & 4.89 & 4.18 & 4.26 & 75.6 & 80.3 & 97.9 & 83.5 & 85.3 \\
\hline & 50.0 & 50.0 & 50.0 & 50.0 & 50.0 & 42.3 & 39.2 & 46.0 & 48.4 & 44.8 & 84.0 & 78.4 & 92.0 & 96.8 & 89.7 \\
\hline \multirow[t]{3}{*}{ Sample 2} & 0 & 0 & 0 & 0 & 0 & $\mathrm{ND}$ & ND & ND & ND & $\mathrm{ND}$ & & & & & \\
\hline & 5.0 & 5.0 & 5.0 & 5.0 & 5.0 & 4.24 & 4.04 & 4.91 & 4.73 & 4.70 & 84.8 & 80.7 & 98.2 & 94.6 & 94.0 \\
\hline & 50.0 & 50.0 & 50.0 & 50.0 & 50.0 & 49.2 & 47.4 & 48.9 & 49.7 & 44.6 & 98.5 & 94.7 & 97.9 & 99.4 & 89.1 \\
\hline \multicolumn{16}{|l|}{ Juice } \\
\hline \multirow[t]{3}{*}{ Sample 1} & 0 & 0 & 0 & 0 & 0 & ND & $\mathrm{ND}$ & $\mathrm{ND}$ & $\mathrm{ND}$ & $\mathrm{ND}$ & & & & & \\
\hline & 5.0 & 5.0 & 5.0 & 5.0 & 5.0 & 4.25 & 4.11 & 5.12 & 4.10 & 4.59 & 85.0 & 82.3 & 102 & 81.9 & 91.8 \\
\hline & 50.0 & 50.0 & 50.0 & 50.0 & 50.0 & 47.5 & 49.5 & 50.8 & 52.8 & 50.2 & 95.1 & 99.1 & 102 & 106 & 100 \\
\hline \multirow[t]{3}{*}{ Sample 2} & 0 & 0 & 0 & 0 & 0 & $\mathrm{ND}$ & $\mathrm{ND}$ & ND & $\mathrm{ND}$ & $\mathrm{ND}$ & & & & & \\
\hline & 5.0 & 5.0 & 5.0 & 5.0 & 5.0 & 4.56 & 4.57 & 4.08 & 4.50 & 4.71 & 91.1 & 91.3 & 81.6 & 90.0 & 94.2 \\
\hline & 50.0 & 50.0 & 50.0 & 50.0 & 50.0 & 54.5 & 52.6 & 54.0 & 57.0 & 55.6 & 109 & 105 & 108 & 114 & 111 \\
\hline
\end{tabular}

ND: not detected.

\section{3 结论}

本研究制备了基于聚(3-丙烯酰胺苯嗍酸-二乙 烯基苯) 多孔整体材料的硼亲和吸附剂并作为 SCSE 的萃取介质。由于萃取介质可与苯并咪唑类 化合物产生包括 B-N 配合、氢键、疏水等在内的多 重作用力, 因此 SCSE-APBDVB 可对 5 种 BUPs 化 合物进行直接有效萃取。在此基础上,建立了可对 实际水样和果汁样品中 BUPs 残留进行有效监测的
SCSE-APBDVB-HPLC/DAD 分离分析方法。研究 结果表明,所建立的方法具有操作简便、灵敏度高、 重现性好和环境友好等特点。

\section{参考文献:}

[1] Hawkins C J, Lavin M F, Parry D L, et al. Anal Biochem, $1986,159(1): 187$

[2] Ding J, Mao L J, Yuan B F, et al. Plant Methods, 2013, 9 (1) $: 1$

[3] Ren L B, Liu Z, Liu Y C, et al. Angew Chem Int Ed, 2009, 
48: 6704

[4] Hu H Y, Zhang Y, Zhang Y, et al. J Chromatogr A, 2014, 1342: 8

[5] Zhou J K, Liu R Y, Song G, et al. Anal Lett, 2009, 42(12): 1805

[6] Zhou J K, Liu R Y, Song G, et al. Food Science and Technology (周建科, 刘瑞英，宋歌，等. 食品科技)，2009，34 (8) : 290

[7] Wang L Z, Zhou Y, Chen Y, et al. Journal of Instrumental Analysis (王连珠, 周昱, 陈泳, 等. 分析测试学报), 2010, 29(3) : 289

[8] Zhou Q X, Zhang X G. J Sep Sci, 2010, 33: 3734

[9] Zhou Q X, Zhang X G, Xie G H. J Sep Sci, 2013, 36: 2323

[10] Zhang J H, Li M, Yang M Y, et al. J Chromatogr A, 2012,
1254: 23

[11] Martinez D B, Galera M M, Vazquez P P, et al. Chromatographia, 2007, 66(3): 533

[12] Huang X J, Chen L L, Lin F H, et al. J Sep Sci, 2011, 34 : 2145

[13] Huang X J, Chen L L, Yuan D X, et al. J Chromatogr A, 2012, 1248: 67

[14] Huang X J, Chen L L, Yuan D X. J Hazard Mater, 2013, 262: 161

[15] Neng N R, Pinto M L, Pires J, et al. J Chromatogr A, $2007,1171(1 / 2): 8$

[16] Huang X J, Qiu N N, Yuan D X, et al. Talanta, 2009, 78 (1) : 101

\section{《岩矿测试》2015 年征订启事}

国内统一刊号：CN11-2131/TD

国际 CODEN： YACEEK

国外发行代号：BM4089
国际标准刊号： ISSN 0254-5357

国内邮发代号: $2-313$ 京西工商广字第 0227 号

《岩矿测试》1982 年创刊, 是中国地质学会岩矿测试技术专业委员会和国家地质实验测试中心共同主办 的学术期刊。是中文核心期刊 ( “地质学” 类), 中国科技核心期刊, 中国期刊方阵双效期刊。已被《化学文 摘》、《文摘杂志》、《剑桥科学文摘》、《乌利希期刊指南》、《皇家化学学会》系列文摘的《分析文摘》和《质谱 学通报-增补》、《哥白尼索引》, 《中国科学引文数据库》 $(\mathrm{CSCD}) 、 《 中$ 国期刊网》( CNKI)、《中文科技期刊全 文数据库》、《万方数据一一科技化期刊群》等 19 家国内外数据库和文摘收录。

《岩矿测试》以国家需求为导向, 坚持地质实验技术创新、面向应用、服务基层的方针和基本定位。以发 表优秀的地质与地球化学分析研究成果为核心目标, 报道国内外地质科学、环境保护、石油化工、冶金及相关 领域的基础性、前瞻性和创新性研究成果, 推动分析测试技术的进步。我刊要求文章的可读性强, 引用价值 高; 注重学术成果的参考价值,追求技术创新方法实用, 研究思路和写作内涵能够给读者启迪与借鉴。

《岩矿测试》投稿网站 http://www.ykcs.ac.cn, 稿件采编自动化,信息发布实时化,过刊全文可免费下 载。

《岩矿测试》为双月刊, 大 16 开, 逢单月上旬出版; 国内外公开发行。2015 年定价 30.0 元/本, 全年 180.0 元。漏订的读者可直接与编辑部联系。

\section{欢迎订阅欢迎投稿欢迎刊登广告}

通讯地址: 北京市西城区百万庄大街 26 号 国家地质实验测试中心(邮政编码 100037)

电话: ( 010$) 68999562$

传真: ( 010$) 68999563$

E-mail: ykcs_zazhi@163.com 